\title{
HEURISTICS FOR THE BRAUER-MANIN OBSTRUCTION FOR CURVES
}

\author{
BJORN POONEN
}

\begin{abstract}
We conjecture that if $C$ is a curve of genus $>1$ over a number field $k$ such that $C(k)=\emptyset$, then a method of Scharaschkin (equivalent to the Brauer-Manin obstruction in the context of curves) supplies a proof that $C(k)=\emptyset$. As evidence, we prove a corresponding statement in which $C\left(\mathbb{F}_{v}\right)$ is replaced by a random subset of the same size in $J\left(\mathbb{F}_{v}\right)$ for each residue field $\mathbb{F}_{v}$ at a place $v$ of good reduction for $C$, and the orders of Jacobians over finite fields are assumed to be smooth (in the sense of having only small prime divisors) as often as random integers of the same size. If our conjecture holds, and if Shafarevich-Tate groups are finite, then there exists an algorithm to decide whether a curve over $k$ has a $k$-point, and the Brauer-Manin obstruction to the Hasse principle for curves over the number fields is the only one.
\end{abstract}

\section{Setup}

Let $k$ be a number field. Fix an algebraic closure $\bar{k}$ of $k$, and let $G=\operatorname{Gal}(\bar{k} / k)$. Let $C$ be a curve of genus $g$ over $k$. (In this paper, curves are assumed to be smooth, projective, and geometrically integral.) Let $\bar{C}=C \times_{k} \bar{k}$. Let $J$ be the Jacobian of $C$, which is an abelian variety of dimension $g$ over $k$. Assume that $\bar{C}$ has a $G$-invariant line bundle of degree 1 : this gives rise to a $k$-morphism $C \rightarrow J$, and it is an embedding if $g>0$. Let $S_{C}$ be the set of finite primes $v$ of good reduction for $C$. Similarly define $S_{A}$ for any abelian variety $A$ over $k$. We have $S_{C} \subseteq S_{\text {J }}$.

\section{Determining the Set of RAtional Points}

Suppose that generators of the Mordell-Weil group $J(k)$ are known. Then $C(k)$ equals the set of points in $J(k)$ that lie on the subvariety $C$. We would like to know whether $C(k)$ can be calculated, especially in the case $g>1$ in which $C(k)$ is guaranteed to be finite by [Faltings1983].

If $J(k)$ is finite, then in principle we can list its elements and check which of them lie on $C$. On the other hand, if $J(k)$ is infinite, it can be very difficult to decide which points of $J(k)$ lie on $C$.

\section{Chabauty's approach}

One approach, due to C. Chabauty [Chabauty1941], works in $J\left(k_{v}\right)$, where $k_{v}$ is the completion of $k$ at a nonarchimedean place $v$. Chabauty observed that the closure $\overline{J(k)}$ of

Date: July 9, 2005.

2000 Mathematics Subject Classification. Primary 11G30; Secondary 11G10, 14 G05.

Key words and phrases. Chabauty, Jacobian, Brauer-Manin obstruction, Hasse principle.

This research was supported by NSF grant DMS-0301280 and a Packard Fellowship. The author thanks the Institut Henri Poincaré and the Isaac Newton Institute for their hospitality. 
$J(k)$ in $J\left(k_{v}\right)$ is an analytic submanifold of $J\left(k_{v}\right)$, and proved that if the rank of $J(k)$ is less than $g$, the subset of points in $\overline{J(k)}$ lying on $C$ is finite; in this case, as explained by R. Coleman [Coleman1985chabauty], one gets an effective upper bound for $\# C(k)$. Often one can even determine $C(k)$ explicitly. But the dimension hypothesis is not always satisfied, and even when it is, the upper bound on $\# C(k)$ may fail to be sharp.

\section{ScharaschKin's APPROACH}

A more recent approach, suggested by V. Scharaschkin [Scharaschkin2004preprint], tries to find which points of $J(k)$ lie on $C$ modulo $\mathfrak{p}$ for many primes $\mathfrak{p}$. More precisely, he proposes the following method for proving that $C(k)$ is empty. Choose a finite subset $S \subset S_{C}$. Let $\mathbb{F}_{v}$ be the residue field at $v$. Then we have a commutative square

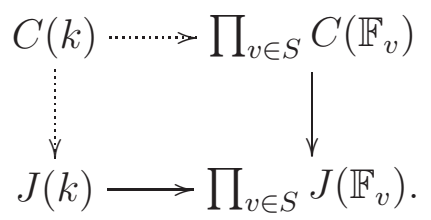

If the images of the solid arrows in $\prod_{v \in S} J\left(\mathbb{F}_{v}\right)$ do not intersect, then $C(k)$ is empty.

Remark 4.1. For this paragraph we assume that the Shafarevich-Tate group $\amalg(J)$ is finite, or at least that its maximal divisible subgroup is trivial. Scharaschkin [Scharaschkin2004preprint] proved that then the potential obstruction to the existence of $k$-points described above is part of the Brauer-Manin obstruction. More precisely, he showed that if we consider the product of $J\left(k_{v}\right)$ (modulo its connected component if $v$ is archimedean) over all places $v$ instead of a product of only $J\left(\mathbb{F}_{v}\right)$ over only places of good reduction, then we recover exactly the Brauer-Manin obstruction.

For the connection of the Brauer-Manin obstruction to the information on rational points obtained from finite étale covers, see [Stoll2005preprint].

\section{A CONJECTURE AND ITS impliCATIONS}

We conjecture the following, based on heuristics to be explained later.

Conjecture 5.1. Let $C \rightarrow J$ be as in Section 1, with $g>1$. If $C(k)=\emptyset$, then there exists a finite subset $S \subset S_{C}$ such that the images of $J(k)$ and $\prod_{v \in S} C\left(\mathbb{F}_{v}\right)$ in $\prod_{v \in S} J\left(\mathbb{F}_{v}\right)$ do not intersect.

The importance of Conjecture 5.1 is given by the following result:

Theorem 5.2. Assume Conjecture 5.1. Assume also that Shafarevich-Tate groups of Jacobians of curves over number fields are finite. Then

(a) There is an algorithm that takes as input a number field $k$ and a curve $C$ over $k$, and decides whether $C$ has k-point.

(b) The Brauer-Manin obstruction to the Hasse principle is the only obstruction to the existence of a $k$-point on a curve $C$ over a number field $k$.

Proof. For details on how elements of $k$ and curves over $k$ can be represented, see [Baker-etal2004preprint].

Before proceeding, we recall a few well-known facts: 
(i) There exists an algorithm for deciding whether a smooth projective variety $X$ over $k$ has a point over every completion $k_{v}$. (Sketch of proof: For all nonarchimedean primes $v$ of sufficiently large norm, the Weil conjectures and Hensel's lemma imply that $X$ automatically has a $k_{v}$-point. One can test the remaining $v$ individually, again using Hensel's lemma in the nonarchimedean case.)

(ii) If $X$ is a torsor of an abelian variety $A$ over a number field $k$, and if $\amalg(A)$ is finite, then there exists an algorithm to decide whether $X$ has a $k$-point, and the BrauerManin obstruction to the Hasse principle is the only one for $X$. (Sketch of proof: By the previous fact, we may assume $X\left(k_{v}\right) \neq \emptyset$ for all $v$, so $X$ represents an element of $\amalg(A)$. If we search for $k$-points on $X$ by day, and perform higher and higher descents on $A$ by night, we will eventually decide whether $X$ has a $k$-point, assuming the finiteness of $\amalg(A)$, even if we are not given a bound on $\# \amalg(A)$. It remains to show that if $X$ has no $k$-point, then there is a Brauer-Manin obstruction. Under our assumption that $\amalg(A)$ is finite, the Cassels-Tate pairing

$$
\langle,\rangle: \amalg(A) \times \amalg\left(A^{\vee}\right) \rightarrow \mathbb{Q} / \mathbb{Z}
$$

is nondegenerate. If $X$ has no $k$-point, then $X$ corresponds to a nonzero element of $\amalg(A)$, so there is a torsor $Y$ of the dual abelian variety $A^{\vee}$ such that $\langle X, Y\rangle \neq 0$. By [Manin1971, Theorem 6], there is an element $y \in \operatorname{Br} X$ related to $Y$ such that the Brauer pairing of $y$ with every adelic point on $X$ gives the value $\langle X, Y\rangle \neq 0$, so $X$ has a Brauer-Manin obstruction.)

We now return to our problem. By [Baker-et-al2004preprint, Lemma 5.1(1)], we can compute the genus $g$ of $C$, so we may break into cases according to the value of $g$.

If $g=0$, then $C$ satisfies the Hasse principle. Thus to test for the existence of a $k$-point, it suffices to use fact (i) above.

If $g=1$, then $C$ is a torsor of its Jacobian $J$, so it suffices to use (ii).

From now on, we suppose $g \geq 2$. By (i), we may reduce to the case that $C\left(k_{v}\right)$ is nonempty for every $v$. Let $X:=\mathbf{P i c}_{C / k}^{1}$ be the variety parametrizing degree-1 line bundles on $C$. Thus $X$ is a torsor of the Jacobian $J$. We have a canonical injection from $C$ to $X$ taking a point $c \in C$ to the class of the associated degree- 1 divisor. By (ii), we can check whether $X$ has a $k$-point; if not, then $C$ has no $k$-point, and there is a Brauer-Manin obstruction for $X$, which pulls back to a Brauer-Manin obstruction for $C$. Thus from now on, we may assume that $X$ has a $k$-point. In other words, $\bar{C}$ has a $G$-invariant line bundle of degree 1 . Such a $G$-invariant line bundle can be found by a search, and it allows us to identify $X$ with $J$. We now can search for $k$-points on $C$ each day, while running Scharaschkin's method using the first $r$ primes in $S_{C}$ for larger and larger $r$ each night. Conjecture 5.1 implies that one of these two processes will terminate. Thus there exists an algorithm for deciding whether $C$ has a $k$-point. Moreover, as mentioned in Remark 4.1, assuming finiteness of $\amalg(J)$, if Scharaschkin's method proves the nonexistence of $k$-points, then there is a Brauer-Manin obstruction.

Remark 5.3. If one knows that the Brauer-Manin obstruction to the Hasse principle is the only one for a smooth projective variety $X$, that in itself lets one determine whether $X$ has a $k$-point, in principle, as we will explain in the following paragraph. This gives an alternative approach to Theorem 5.2(a), based on part (b). 
By an unpublished result of O. Gabber, reproved by A. J. de Jong, each element of the cohomological Brauer group $\operatorname{Br} X:=H_{\text {et }}^{2}\left(X, \mathbb{G}_{m}\right)$ can be represented by an Azumaya algebra $\mathcal{A}$, i.e., a locally free $\mathcal{O}_{X}$-algebra that is étale locally isomorphic to a finite-dimensional matrix algebra. Each $\mathcal{A}$ can be described by a finite amount of data:

(1) a covering of $X$ by finitely many Zariski open subsets $X_{i}$ such that $\left.\mathcal{A}\right|_{X_{i}}$ is free as an $\mathcal{O}_{X_{i}}$-module,

(2) the multiplication table for $\left.\mathcal{A}\right|_{X_{i}}$ with respect to a chosen $\mathcal{O}_{X_{i}}$-basis, for each $i$,

(3) the change-of-basis map on the intersection $X_{i} \cap X_{j}$, for each $i$ and $j$,

(4) a covering $U_{i} \rightarrow X_{i}$ in the étale topology, for each $i$,

(5) a positive integer $r_{i}$ and an $\mathcal{O}_{U_{i}}$-algebra isomorphism $\mathcal{A} \otimes_{\mathcal{O}_{X}} \mathcal{O}_{U_{i}} \simeq M_{r_{i}}\left(\mathcal{O}_{U_{i}}\right)$, for each $i$.

Moreover, given such data, one can easily check whether it actually defines an Azumaya algebra. Therefore there is a (very inefficient) algorithm which when left running forever, eventually produces all Azumaya algebras over $X$, each possibly occurring more than once, simply by enumerating and checking each possible set of data. Now, we search for $k$-points by day and generate Azumaya algebras by night, calculating at the end of each night whether the Azumaya algebras generated so far give an obstruction.

Remark 5.4. It is not clear whether Conjecture 5.1 and the finiteness of Shafarevich-Tate groups imply the existence of an algorithm for listing all $k$-points on a given curve $C$ of genus $g \geq 2$ over $k$. For the listing problem, applying Chabauty's method to finite étale covers seems more promising: see [Stoll2005preprint] for an analysis of the situation.

\section{Computational evidence For the COnjecture}

E. V. Flynn [Flynn2004] has developed an implementation of Scharaschkin's method for genus-2 curves over $\mathbb{Q}$. He tested 145 such curves defined by equations with small integer coefficients, having $\mathbb{Q}_{p}$-points for all $p \leq \infty$, but having no $\mathbb{Q}$-point with $x$-coordinate of height less than $10^{30}$. These were grouped according to the rank $r$ of the Jacobian. In all cases with $r \leq 1$, he successfully showed that there was a Brauer-Manin obstruction. In most cases with $r=2$, a Brauer-Manin obstruction was found, and all the unresolved $r=2$ cases were later resolved by an improved implementation of M. Stoll. The remaining cases had $r=3$, and a few of these were resolved; it was unclear from the computation whether the remaining ones could be resolved by a longer computation: the combinatorics quickly became prohibitive.

\section{TheORETICAL EVIDENCE FOR THE CONJECTURE}

Here we give a heuristic analysis of Conjecture 5.1.

Recall that if $B \in \mathbb{R}_{>0}$, an integer is called $B$-smooth if all its prime factors are $\leq B$. For any fixed $u \in(0,1)$, the fraction of integers in $[1, B]$ that are $B^{u}$-smooth tends to a positive constant as $B \rightarrow \infty$ [DeBruijn1951].

As our main evidence for Conjecture 5.1, we prove a modified version of it in which $C\left(\mathbb{F}_{v}\right)$ is modelled by a random subset of $J\left(\mathbb{F}_{v}\right)$ of the same order, and in which we assume that the integer $\# J\left(\mathbb{F}_{v}\right)$ is as smooth as often as a typical integer of its size. The smoothness assumption is formalized in the following: 
Conjecture 7.1. Let $A$ be an abelian variety over a number field $k$, and let $u \in(0,1)$. Then

$$
\limsup _{B \rightarrow \infty} \frac{\left\{v \in S_{A}: \# \mathbb{F}_{v} \leq B \text { and } \# A\left(\mathbb{F}_{v}\right) \text { is } B^{u} \text {-smooth }\right\}}{\left\{v \in S_{A}: \# \mathbb{F}_{v} \leq B\right\}}>0 .
$$

Let $g=\operatorname{dim} A$. If $\# A\left(\mathbb{F}_{v}\right)$ behaves like a typical integer of its size, which is about $\left(\# \mathbb{F}_{v}\right)^{g} \leq B^{g}$, then it should have a positive probability of being $B^{u}$-smooth, since $B^{u}$ is a constant power of $B^{g}$. If anything, $\# A\left(\mathbb{F}_{v}\right)$ can be expected to factor more than typical integers its size, because of splitting of $A$ up to isogeny, or because of biases in the probability of being divisible by small primes. Thus Conjecture 7.1 is reasonable.

We are now ready to state our main result giving evidence for Conjecture 5.1.

Theorem 7.2. Let $C \rightarrow J$ be as in Section 1, with $g>1$. Assume Conjecture 7.1 for $J$. For each prime $v \in S_{C}$, let $\mathcal{C}\left(\mathbb{F}_{v}\right)$ be a random subset of $J\left(\mathbb{F}_{v}\right)$ of size $\# C\left(\mathbb{F}_{v}\right)$; we assume that the choices for different $v$ are independent. Then with probability 1 , there exists a finite subset $S \subseteq S_{C}$ such that the images of $J(k)$ and $\prod_{v \in S} \mathcal{C}\left(\mathbb{F}_{v}\right)$ in $\prod_{v \in S} J\left(\mathbb{F}_{v}\right)$ do not intersect.

Proof. It suffices to find $S$ such that the probability that the images intersect is arbitrarily small.

Given $B>0$, let $S=S(B)$ be the set of $v \in S_{C}$ such that $\# \mathbb{F}_{v} \leq B^{2}$ and $\# J\left(\mathbb{F}_{v}\right)$ is $B$-smooth. Because we have assumed Conjecture 7.1 for $J$, there exists $c>0$ such that for arbitrarily large $B \in \mathbb{R}_{>0}$ (the square root of the $B$ occurring in Conjecture 7.1), the set $S$ contains at least a fraction $c$ of the primes $v \in S_{C}$ with $\# \mathbb{F}_{v} \leq B^{2}$.

Let $\pi(x)$ be the number of rational primes $\leq x$. The prime number theorem says that $\pi(x)=(1+o(1)) x / \log x$ as $x \rightarrow \infty$. For $v \in S$, the Weil conjectures give $\# J\left(\mathbb{F}_{v}\right) \leq$ $O\left(\left(\# \mathbb{F}_{v}\right)^{2 g}\right) \leq B^{2 g+o(1)}$ as $B \rightarrow \infty$ so by $B$-smoothness, the least common multiple $L$ of $\# J\left(\mathbb{F}_{v}\right)$ for $v \in S$ satisfies

$$
\begin{aligned}
L & \leq \prod_{\operatorname{primes} p \leq B} p^{\left\lfloor\log _{p} B^{2 g+o(1)}\right\rfloor} \\
& \leq \prod_{\operatorname{primes} p \leq B} B^{2 g+o(1)} \\
\log L & \leq \pi(B)(2 g+o(1)) \log B \\
& =(2 g+o(1)) B .
\end{aligned}
$$

Suppose that $J(k)$ is generated by $r$ elements. Every element of $\prod_{v \in S} J\left(\mathbb{F}_{v}\right)$ has order dividing $L$, so the order of the image $I$ of $J(k)$ in $\prod_{v \in S} J\left(\mathbb{F}_{v}\right)$ is at most

$$
L^{r} \leq \exp ((2 g+o(1)) r B)
$$

The probability that a fixed element of a set of size $n$ belongs to a random subset of size $m$ is $m / n$, so the probability $P$ that a fixed element of $I$ belongs to the image $I^{\prime}$ of 


\section{$\prod_{v \in S} \mathcal{C}\left(\mathbb{F}_{v}\right) \rightarrow \prod_{v \in S} J\left(\mathbb{F}_{v}\right)$ satisfies}

$$
\begin{aligned}
P & =\prod_{v \in S} \frac{\# C\left(\mathbb{F}_{v}\right)}{\# J\left(\mathbb{F}_{v}\right)} \\
& \leq \prod_{v \in S} \frac{\left(\# \mathbb{F}_{v}\right)^{1+o(1)}}{\left(\# \mathbb{F}_{v}\right)^{g+o(1)}} \\
\log P & =(1-g+o(1)) \sum_{v \in S} \log \# \mathbb{F}_{v},
\end{aligned}
$$

where again we have used the Weil conjectures. The number of primes $v$ of $k$ with $\# \mathbb{F}_{v} \leq$ $B^{2}$ is at least the number of rational primes $p \leq B^{2}$ that split completely in $k$, which is asymptotically $\geq c_{1} \pi\left(B^{2}\right)$. (We use $c_{1}, c_{2}, \ldots$ to denote positive constants independent of $B$.) Since $S$ contains a positive fraction of these $v$, and since the numbers $\# \mathbb{F}_{v}$ are powers of primes, with each prime occurring at most $[k: \mathbb{Q}]$ times, we find that $\sum_{v \in S} \log \# \mathbb{F}_{v}$ is at least the sum of $\log p$ for the first $c_{2} \pi\left(B^{2}\right)$ primes. By the prime number theorem, the $n$-th prime is $(1+o(1)) n \log n$, so

$$
\begin{aligned}
\sum_{v \in S} \log \# \mathbb{F}_{v} & \geq \sum_{n=1}^{c_{2} \pi\left(B^{2}\right)} \log ((1+o(1)) n \log n) \\
& \geq \sum_{n=c_{2} \pi\left(B^{2}\right) / 2}^{c_{2} \pi\left(B^{2}\right)} \log ((1+o(1)) n \log n) \\
& \geq c_{3} \pi\left(B^{2}\right) \log \pi\left(B^{2}\right) \\
& \geq c_{4} B^{2}
\end{aligned}
$$

as $B \rightarrow \infty$. Since $1-g<0$, we get

$$
\begin{aligned}
\log P & \leq-c_{5} B^{2} \\
P & \leq \exp \left(-c_{5} B^{2}\right) .
\end{aligned}
$$

Thus the probability that $I$ intersects $I^{\prime}$ is at most

$$
\# I \cdot P \leq \exp ((2 g+o(1)) r B) \cdot \exp \left(-c_{5} B^{2}\right),
$$

which tends to 0 as $B \rightarrow \infty$, as desired.

Remark 7.3. For the case where $C(k)$ is nonempty, we would have liked to analyze a refined heuristic that reflects the existence of the $k$-points. Namely, suppose that $\mathcal{C}\left(\mathbb{F}_{v}\right)$ is a random subset of $J\left(\mathbb{F}_{v}\right)$ of size $\# C\left(\mathbb{F}_{v}\right)$ chosen subject to the constraint that it contains the image of $C(k)$ in $J\left(\mathbb{F}_{v}\right)$. We then expect that with probability 1 , the only points of $J(k)$ whose image in $\prod_{v \in S_{C}} J\left(\mathbb{F}_{v}\right)$ lie in the image of $\prod_{v \in S_{C}} \mathcal{C}\left(\mathbb{F}_{v}\right)$ are those in $C(k)$. But we were unable to prove this, even assuming Conjecture 7.1.

Question 7.4. Is it true more generally that if $X$ is a closed subvariety of an abelian variety $A$ over a number field $k$, and $S$ is a density-1 set of primes of good reduction for $X$ and $A$, then the intersection of the closure of the image of $A(k)$ in $\prod_{v \in S} A\left(\mathbb{F}_{v}\right)$ with $\prod_{v \in S} X\left(\mathbb{F}_{v}\right)$ equals the closure of the image of $X(k)$ in $\prod_{v \in S} A\left(\mathbb{F}_{v}\right)$ ? One could also ask the question with $\prod_{v \in S} A\left(\mathbb{F}_{v}\right)$ replaced by the product of $A\left(k_{v}\right)$ (modulo its connected component) over all $v$. 
We expect a positive answer; this together with finiteness of $\amalg(A)$ would imply that the Brauer-Manin obstruction to the Hasse principle is the only one for such $X$ : cf. Remark 4.1.

\section{ACKNOWLEDGEMENTS}

I thank Jean-Louis Colliot-Thélène and Michael Stoll for several discussions about the Brauer-Manin obstruction during the Fall 2004 trimester at the Institut Henri Poincaré.

\section{REFERENCES}

[Baker-et-al2004preprint] Matthew H. Baker, Enrique González-Jiménez, Josep González, and Bjorn Poonen, Finiteness results for modular curves of genus at least 2, 2004, Preprint, arXiv:math.NT/0211394.

[Chabauty1941] Claude Chabauty, Sur les points rationnels des courbes algébriques de genre supérieur à l'unité, C. R. Acad. Sci. Paris 212 (1941), 882-885. MR0004484 (3,14d) (French)

[Coleman1985chabauty] Robert F. Coleman, Effective Chabauty, Duke Math. J. 52 (1985), 765-770. MR808103 (87f:11043)

[DeBruijn1951] N. G. de Bruijn, On the number of positive integers $\leq x$ and free of prime factors $>y$, Nederl. Acad. Wetensch. Proc. Ser. A. 54 (1951), 50-60. MR0046375 (13,724e)

[Faltings1983] G. Faltings, Endlichkeitssätze für abelsche Varietäten über Zahlkörpern, Invent. Math. 73 (1983), 349-366. MR718935 (85g:11026a) (German)

[Flynn2004] E. V. Flynn, The Hasse principle and the Brauer-Manin obstruction for curves, Manuscripta Math. 115 (2004), 437-466. MR2103661

[Manin1971] Y. I. Manin, Le groupe de Brauer-Grothendieck en géométrie diophantienne, Actes du Congrès International Des Mathématiciens (Nice, 1970), Tome 1, Gauthier-Villars, Paris, 1971, pp. 401-411. MR0427322 (55 \#356)

[Scharaschkin2004preprint] Victor Scharaschkin, The Brauer-Manin obstruction for curves (December 2004), Preprint.

[Stoll2005preprint] Michael Stoll, Finite descent and rational points on curves, 2005, Preprint.

Department of Mathematics, University of California, Berkeley, CA 94720-3840, USA

E-mail address: poonen@math.berkeley.edu

$U R L:$ http://math.berkeley.edu/ ${ }^{\sim}$ poonen 